\title{
Evaluation de la photographie aérienne pour l'aide au repérage des foyers de rhizomanie et au diagnostic de la rhizomanie de la betterave à sucre
}

\author{
B. Andrieu \\ INRA, Station de bioclimatologie, 78850 Thiverval-Grignon, France
}

(reçu le 27-9-1988, accepté le 25-1-1989)

Résumé - On évalue l'apport de la photographie aérienne pour superviser les prélèvements destinés au repérage des foyers de rhizomanie. La méthode est fondée sur la mise en évidence par photo-interprétation des chloroses induites par la rhizomanie; les résultats sont évalués à l'aide d'un inventaire réalisé sur le terrain.

L'efficacité de la méthode proposée dépend principalement des conditions climatiques et hydriques, qui déterminent l'intensité des symptômes foliaires. Les résultats portent sur 2 années d'étude: dans les conditions de forte chaleur et d'humidité obtenues la première année, le taux de détection a été de $72 \%$ et aurait probablement été augmenté en améliorant les conditions de prise de vue; en revanche, en cas de sécheresse aux mois d'août et septembre, les symptômes de stress hydrique réduisent considérablement les possibilités de repérage.

Beta vulgaris - chlorose - photographie aérienne - rhizomanie - diagnostic

Summary - Evaluation of aerial photography as and aid to diagnosing rizomantia of sugar beet. The purpose of this study is to evaluate aerial photography as an aid to supervising the ground investigations necessary to the diagnosis of rizomania. The method is based on photo-interpretation to detect chlorosis due to rizomania. Results are for 2 years of study; accuracy of the methods depends strongly on climatic and hydric conditions, that determine the intensity of foliar symptoms: with hot and wet weather conditions occuring the first year, $72 \%$ of infested plots could be detected and this figure would probably have been increased by improvments in the acquisition of data. The second year, a drought occured in august and september, and hydric stress reduced strongly the possibility of diagnosing rizomania.

Beta vulgaris - chlorosis - aerial photography - rizomania - diagnosis

\section{Introduction}

La rhizomanie de la betterave est une maladie attachée au sol, liée à la présence du virus des nervures jaunes et nécrotiques de la betterave (BNYVV) et du champignon Polymyxa betae Keskin. II n'existe pas de méthodes de traitement curatif et les chutes de rendement $(50 \%$ et plus) entraînent l'abandon progressif de la culture dans les zones atteintes. Les méthodes de lutte s'appuient essentiellement sur la recherche et l'amélioration de variétés tolérantes et sur des mesures prophylactiques destinées à ralentir la progression de la maladie (Putz et Richard Molard, 1984a; Cariolle, 1987).

Le repérage des zones atteintes détermine l'efficacité des mesures prophylactiques et per- met de décider de l'utilisation éventuelle de variétés tolérantes; un diagnostic fiable ne peut être obtenu que par l'observation des racines et les tests sérologiques, ce qui pose le problème du choix des parcelles et de la localisation dans la parcelle des prélèvements à effectuer. Ce choix s'appuie sur des connaissances a priori (persistance de la maladie dans le sol, contamination par le transport de terre ou de boue) et sur l'observation de symptômes foliaires, qui sont peu spécifiques, mais constituent dans la pratique le seul moyen d'identifier une parcelle nouvellement infestée.

Le travail présenté vise à évaluer les possibilités de la télédétection pour la mise en évidence des décolorations foliaires induites par la rhizomanie. Une résolution spatiale élevée est néces- 
saire pour la détection des débuts d'atteintes, ce qui nous a conduit à utiliser la photographie aérienne. L'efficacité d'une telle approche dépend de plusieurs facteurs:

- relation entre la rhizomanie et l'apparition de chloroses;

- fréquence d'occurence d'autres sources de chloroses (carence, jaunisse);

- aptitude du système utilisé à détecter des variations de coloration foliaire et à les différencier des variations de réflectance liées à la structure du couvert (port des plantes, indice foliaire).

L'étude porte sur 2 années (1983 et 1985) très contrastées sur le plan climatique.

Les clichés sont analysés par photo-interprétation et les résultats sont évalués à l'aide d'un inventaire réalisé sur le terrain.

\section{Matériel et Méthodes}

\section{Symptômes foliaires}

La rhizomanie est susceptible de se manifester par des modifications dans le port et la pigmentation foliaire (Sanwald, 1981; Asher, 1985; Anonyme, 1984; Putz et Richard Molard, 1984b).

\section{1) Port de la plante}

La rhizomanie perturbe le système racinaire, et donc l'alimentation hydrique de la plante, et peut provoquer un flétrissement anormal du feuillage. La quasi-totalité des parcelles présente un flétrissement au milieu des journées chaudes, aussi celui-ci ne constitue un élément de diagnostic que s'il intervient dans des conditions particulières (début de journée, ciel couvert, pluie récente, etc.), ce qui le rend difficile à utiliser par télédétection.

\section{2) Décolorations foliaires}

Le diagnostic aérien et l'inventaire au sol se fondent principalement sur un jaunissement du feuillage analogue à celui provoqué par une carence; on préserıte ici l'ordre de grandeur des variations de réflectance induites par la rhizomanie et les problèmes liés à l'utilisation de ce symptôme.

a) Ordre de grandeur. Des mesures réalisées au spectrophotomètre montrent que la réflectance foliaire de plantes malades peut atteindre 0,30 à 0,40 dans le visible, contre moins de 0,10 pour une feuille saine (Fig. 1).

Les variations de réflectance $(\rho)$ induites au niveau du couvert ont été évaluées en utilisant d'une part un radiomètre Cimel, simulant les bandes du satellite SPOT, et d'autre part des mesures densitométriques sur des clichées acquis à une altitude de $300 \mathrm{~m}$, pour laquelle on peut négliger l'effet de l'atmosphère sur les mesures. Afin de réaliser des mesures les plus comparables possibles sur couvert sain et atteint, on s'est intéressé aux variations de réflectance observables aux transitions entre des zones saines et des zones présentant des décolorations foliaires marquées (notation 2,5 sur une échelle de 0 à 4 par un observateur sur le terrain).

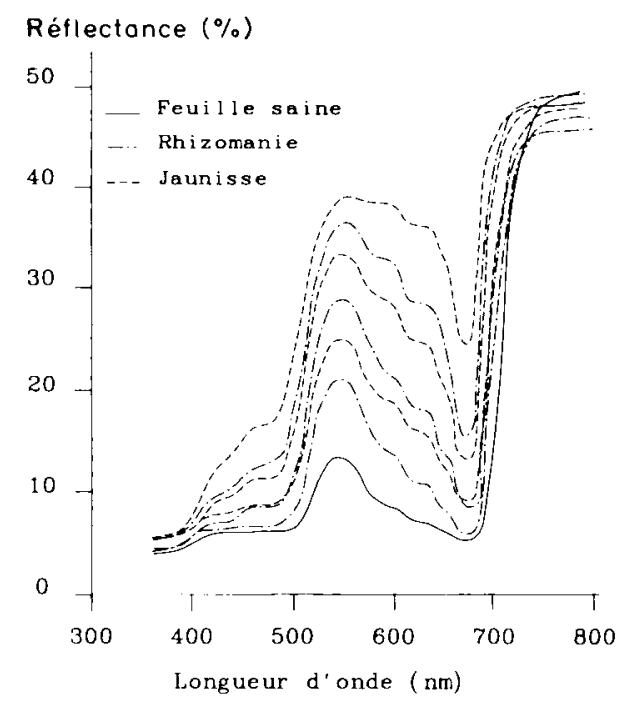

Fig. 1. Réflectance de feuilles de betteraves atteintes de jaunisse ou de rhizomanie.

Les mesures radiométriques (Tableau l) ont été réalisées sur des couverts aussi comparables que possible sur le plan du taux de couverture au sol, toutefois, le couvert atteint est très légèrement moins couvrant que le couvert sain, ce qui explique les différences de réflectance observées dans le proche infrarouge (qui restent toutefois non significatives) et accentue les écarts observés dans le visible. Les différences de réflectance entre couvert sain et décoloré sont de l'ordre de 0,02 dans le vert et de 0,01 dans le rouge. Ces valeurs sont faibles dans l'absolu, mais correspondent à des variations relatives $(\Delta \rho / \rho)$ de l'ordre de $25 \%$.

Le film photographique est bien adapté à la mise en évidence des variations intervenant sur des cibles à faible niveau de réflectance, car la densité de l'émulsion varie comme le logarithme de l'énergie reçue:

Dans le cas d'une émulsion couleur on a pour chaque couche du film:

$$
D=D o-\gamma \log _{10}(E)
$$

- D est la densité analytique de la couche considérée, - Do et $\gamma$ sont deux constantes dépendant du film utilisé et des conditions de développement;

- E est l'exposition, produit de l'énergie reçue par la sensibilité de l'émulsion.

La densitométrie trichrome permet de mesurer la densité optique de l'émulsion dans les bandes spectrales d'absorption des colorants cyan, magenta et jaune. Les densités ainsi mesurées ne sont pas rigoureusement les densités analytiques de l'équation (1), car il existe un certain recouvrement des domaines d'absorption des pigments; nous avons négligé cet effet du second ordre pour le calcul suivant, qui vise à établir un ordre de grandeur.

Les relations entre l'exposition $E$ et la réflectance de la cible ont été étudiées par différents auteurs, dont, récemment, Becker et al., 1988. Le problème pris dans son intégralité est complexe du fait en particulier de la réflectance atmosphérique et des variations de certains paramètres en fonction de l'angle de 
Tableau I. Modifications de réflectance du couvert induites par des décolorations foliaires. \%C: couverture du sol (évaluée visuellement); Rv: réflectance dans la bande $500 \mathrm{~nm}-600 \mathrm{~nm}$ (\%); Rr: réflectance dans la bande $600 \mathrm{~nm}-$ $700 \mathrm{~nm}(\%)$; Rir: réflectance dans la bande $800 \mathrm{~nm}-900 \mathrm{~nm}(\%)$; Chaque valeur est la moyenne de 10 à 15 mesures réalisées au voisinage du midi solaire, en l'absence de flétrissement perceptible du feuillage.

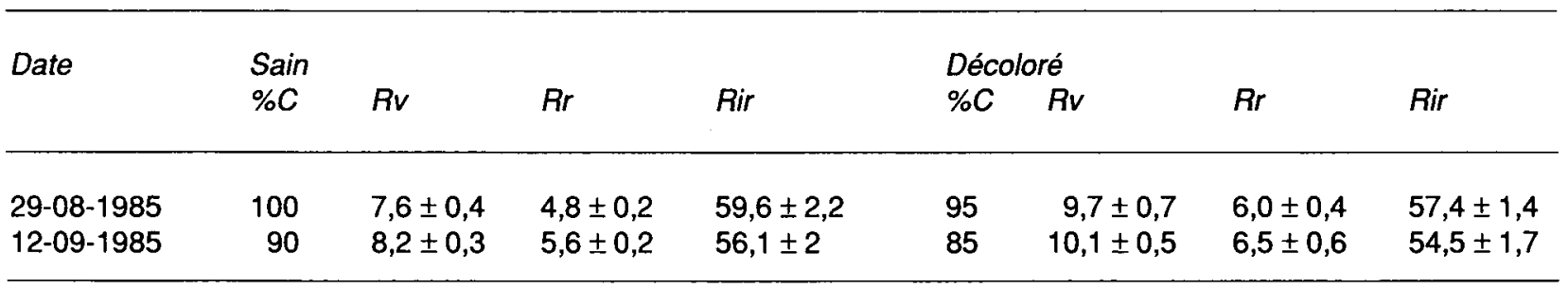

visée; toutefois, lorsque l'effet de l'atmosphère peut être négligé, et pour une inclinaison donnée de la visée, $\mathrm{E}$ est proportionnel à la réflectance de la cible. La différence de densité entre deux points voisins (1 et 2), sur un cliché acquis à basse altitude, est donc liée aux variations de réflectance de la cible par:

$$
D_{2}-D_{1}=-\gamma \log _{10}\left(\rho_{2} / \rho_{1}\right)
$$

On a mesuré pour 3 sites, correspondant à des couverts totalement couvrants, les modifications de densités à la transition entre une zone saine et une zone atteinte, sur des clichés acquis à $300 \mathrm{~m}$ d'altitude avec une émulsion couleur Aérochrome MS 2448. Les décolorations foliaires affectent de façon très voisine les couches cyan et magenta, sensibles respectivement au rouge et au vert; les différences de densité entre zone saine et zone atteinte varient de 0,20 à 0,30 UDO (unité de densité optique), ce qui est largement supérieur à la précision de mesure $( \pm 0,01$ UDO). En prenant pour $\gamma$ la valeur 2,8 calculée à partir de la fiche technique fournie par le fabricant, l'équation (2) permet de calculer la variation relative de réflectance du couvert: $(\Delta \rho / \rho)=18 \%$ à $28 \%$ pour $\Delta D=0,20$ à 0,30 UDO; les valeurs ainsi estimées sont donc comparables à celles obtenues par radiométrie de terrain.

Les ordres de grandeur calculés montrent que les modifications de réflectance du couvert, correspondant à des décolorations foliaires modérées à fortes, sont à peine supérieures à l'incertitude de mesures obtenues par radiométrie de terrain; toutefois, les variations obtenues dans le visible sont compatibles avec la sensibilité d'une émulsion photographique, surtout lorsque I'on peut travailler de façon différentielle (comparaison zone saine/zone atteinte).

b) Limites inhérentes à un diagnostic fondé sur les décolorations foliaires. L'utilisation des décolorations foliaires pour l'inventaire des atteintes pose plusieurs difficultés:

- le jaunissement n'est pas systématique: les décolorations sont très spectaculaires lorsque l'été est chaud et humide, surtout en cultures irriguées, alors qu'elles sont faibles ou inexistantes en conditions sèches.

- ce type de décoloration n'est pas spécifique à la rhizomanie, des confusions sont possibles avec des décolorations liées à des carences, à la sénescence et parfois à la jaunisse. Sur le terrain, ces confusions peuvent généralement être levées par l'observation de la racine, ou par le test ELISA réalisé au laboratoire;

- les symptômes évoluent dans le temps: en 1983 on a observé sur notre site d'étude une généralisation progressive des symptômes au cours du mois d'août et au début de septembre; en 1985, on a observé une évolution comparable au mois d'août puis, compte tenu de l'arrêt des irrigations (vers le 15 août) et de l'absence de précipitations, une stagnation ou une réduction des symptômes en septembre. Ces deux évolutions contrastées ont également été observées par Hansi (1987) et Sanwald (1981) respectivement.

\section{Site d'étude}

Le site d'étude est situé à proximité de Pithiviers (Loiret), dans un secteur partiellement irrigué. Les missions aériennes réalisées en 1983 couvrent 2000 ha, comprenant 110 parcelles de betterave, de dimensions généralement comprises entre 1 ha et 3 ha (Fig. 2a). Cette zone a été étendue en 1985, de façon à inclure une région à plus grand parcellaire ( 3 ha à 20 ha, Fig. 2b) présentant un taux d'infestation moins élevé. Au total, la zone étudiée en 1985 couvre 6000 ha et comprend 230 parcelles.

\section{1) Conditions climatiques (Fig. 3)}

L'année 1983 se caractérise par des températures et des précipitations estivales élevées, conditions très favorables au développement de la maladie et à l'extériorisation des symptômes foliaires, y compris en culture sèche (ITB, 1983). L'année 1985 se caractérise par des températures élevées en septembre et surtout des précipitations très faibles aux mois d'août et septembre d'où des symptômes atténués, voire inexistants en zone non irriguée, et une incidence limitée de la maladie sur le rendement (ITB, 1985).

\section{2) Taux d'atteintes et taux d'irrigation}

Le tableau II donne les taux d'atteinte en cultures sèches et irriguées pour les 2 années d'étude. Le taux d'atteinte est beaucoup plus élevé en 1983 qu'en 1985 , surtout en cultures sèches. Cette différence s'explique d'une part par les conditions climatiques et, d'autre part, par le fait que le site retenu en 1983 correspond au centre du foyer d'infestation, alors qu'en 1985 , on a pris en compte un grand nombre de parcelles situées en périphérie du foyer.

\section{Inventaire au sol}

\section{1) Méthode}

L'inventaire au sol se fonde sur le repérage visuel des symptômes foliaires, complété par l'observation des racines et, en cas de doute, par des tests ELISA. On utilise la cartographie des atteintes mise à notre disposition par l'Institut technique de la betterave industrielle, complétée par nos propres observations: chaque parcelle est ainsi inspectée 4 à 5 fois au cours des 


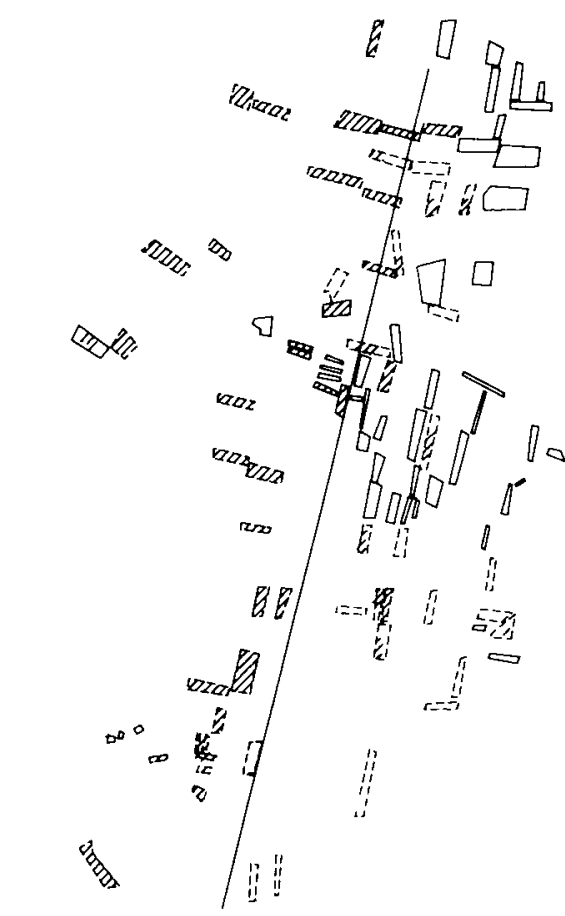

a

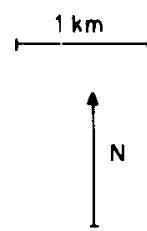

b

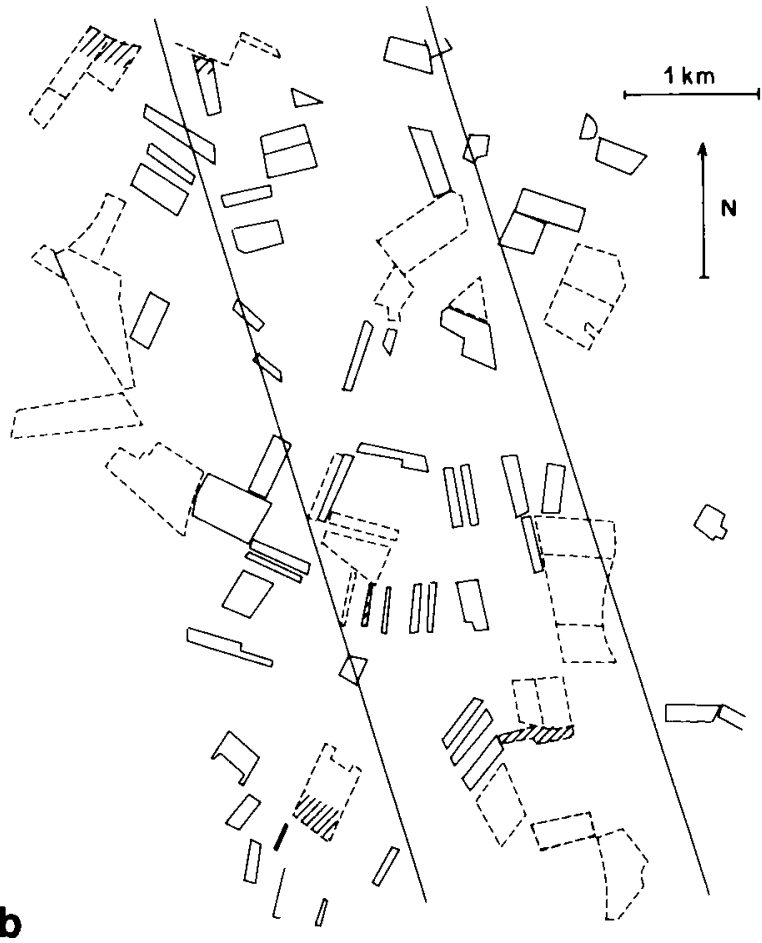

Fig.2. Représentation partielle du site d'étude. La Figure 2a est représentative du site étudié en 1983. Ce site a été étendu en 1985 en incluant une région à plus grand parcellaire (Fig. 2b). - betteraves non irriguées; - - - betteraves irriguées; //// atteintes de rhizomanie.
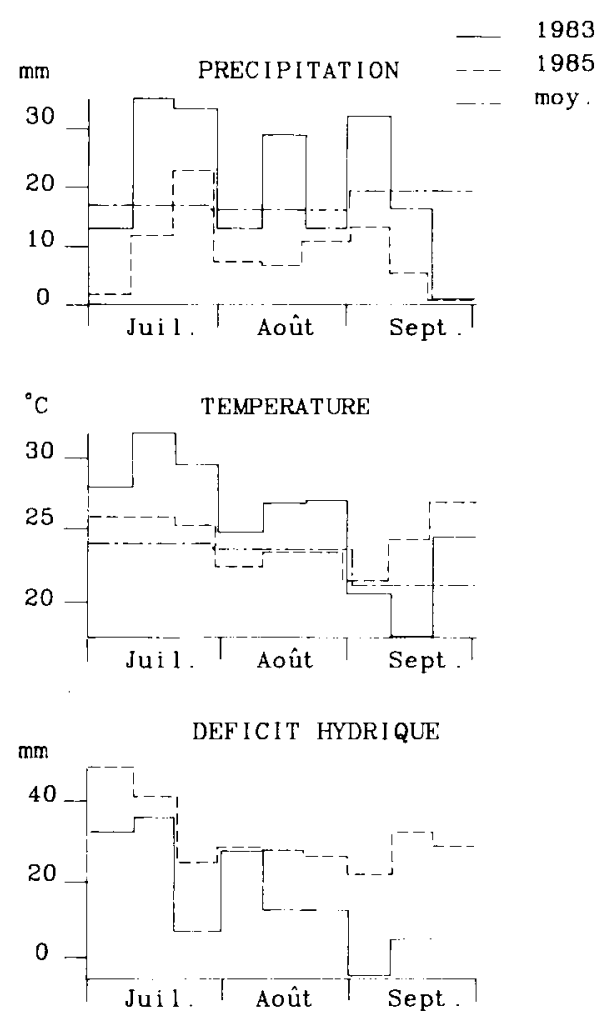

Fig. 3. Conditions climatiques: $1983-1985$ et moyennes: 1954-1981. Précipitations: cumul décadaire; température: moyenne décadaire des températures journalières maximales; déficit hydrique: cumul décadaire. mois d'août et septembre, période au cours de laquelle les symptômes évoluent fortement.

Compte tenu de la persistance de la maladie dans le sol et de sa propagation par le transport de terre, l'inventaire s'appuie également sur des connaissances a priori: présence de la rhizomanie dans une autre parcelle de la même exploitation, observation de la rhizomanie les années précédentes, etc.

Lors des contrôles de terrain, on note approximativement l'intensité et l'extension des symptômes, toutefois ces informations n'ont qu'une valeur indicative, du fait de l'évolution des atteintes dans le temps. Pour l'évaluation des documents aériens, on a retenu une notation en 2 classes seulement (sain/atteint). Une parcelle est classée atteinte si la rhizomanie a été observée au moins une fois, indépendamment de l'intensité et de l'extension des symptômes et de la date d'observation.

\section{2) Fiabilité de l'inventaire au sol}

L'observation visuelle constitue un élément primordial de l'enquête de terrain. Une évaluation du diagnostic visuel au niveau de la plante (observation du feuillage et des racines) a été réalisée par l'ITB en 1985 sur un lot de 213 échantillons provenant de différentes régions (ITB, 1985).

Pour 89 échantillons (42\%), le diagnostic visuel était incertain, toutefois, ce dernier chiffre n'est pas représentatif de la proportion existant sur le terrain, car ce sont les échantillons douteux qui ont fait prioritairement l'objet de cette analyse. Lorsqu'il est possible, le diagnostic visuel semble fiable, en effet, sur les 124 échantillons restants: 
Tableau II. Caractérisation du site d'étude.

\section{Année}

1983

Nombre de parcelles étudiées

Taux d'irrigation

Taux d'atteinte (global)

Taux d'atteinte en non irrigué

Taux d'atteinte en irrigué
109

$81 \%(88 / 109)$

$86 \%(94 / 109)$

$67 \%(14 / 21)$

$91 \%(80 / 88)$
228

$60 \%(137 / 228)$

$36 \%(82 / 228)$

$5 \%(5 / 91)$

$56 \%(77 / 137)$
- 99\% (96 sur 97) des échantillons au diagnostic visuel positif étaient positifs au test ELISA;

- 96\% (26 sur 27) des échantillons au diagnostic visuel négatif étaient négatifs au test ELISA.

En ce qui concerne la cartographie des atteintes, if faut noter que l'observation des racines, qui est un élément important du diagnostic visuel, n'a lieu sur le terrain que pour les plantes ayant attiré l'attention par des symptômes foliaires. Des omissions peuvent donc intervenir lors d'atteintes localisées ou de symptômes peu marqués. II est possible que l'inventaire au sol sous-estime les surfaces atteintes en culture non irriguée, tout au moins en année sèche.

\section{Photographies aériennes}

\section{1) Conditions de prise de vue}

On présente ici les résultats obtenus dans les conditions suivantes:

- émulsion couleur visible Aérochrome 2448; format $240 \mathrm{~mm}$;

- focale $150 \mathrm{~mm}$; altitude $1500 \mathrm{~m} / \mathrm{sol}$; échelle 1/10000; recouvrement entre clichés: $60 \%$;

- dates: 13 août et 7 septembre 1983; 29 août et 12 septembre 1985.

La qualité des prises de vue est moyenne en 1983, en particulier du fait de la présence de cumulus lors de la mission du 7 septembre. Elle est très bonne pour les deux missions de 1985.

\section{2) Analyses des clichés}

Certains clichés aériens ont été analysés par microdensitométrie trichrome, afin d'évaluer une procédure automatique d'aide au diagnostic se fondant sur une classification d'après les valeurs radiométriques des pixels, après correction des variations liées à l'angle de visée (Andrieu, 1984). Toutefois l'utilisation pratique de cette technique est limitée par:

- le volume de données à traiter: la numérisation d'un seul cliché avec une résolution métrique (i.e. comparable à celle obtenue par photo-interprétation) représente un volume de données de $3 \cdot 10^{7}$ octets, soit presque la moitié de celui d'une scène SPOT;

- les imprécisions dans la méthode de correction des effets angulaires;

- la non-prise en compte de certains critères utilisables en photo-interprétation (forme des taches, taux de couverture du sol).

La Figure 4 montre que les variations de densité optique sur les clichés, aux transitions entre zones saines et zones atteintes, se manifestent de façon analogue sur les couches sensibles au rouge et au vert et peuvent atteindre 0,2 à 0,3 UDO, comme c'était le cas pour les photographies acquises à basse altitude.

L'analyse globale des missions a été réalisée par photo-interprétation. Les décolorations liées à la rhizomanie se traduisent sur les clichés aériens par une teinte vert pâle ou jaune (Fig. 5). Du fait des variations liées à l'angle de visée, la couleur est appréciée essentiellement par contraste avec les zones voisines, en outre, seule une partie de chaque cliché peut être utilisée.

Les confusions peuvent provenir des différents facteurs modifiant la coloration foliaire ou la structure du couvert. Le choix de la période de prise de vue et l'utilisation de critères relatifs à la forme des taches et au taux de couverture du sol permettent de réduire les risques d'erreur.

a) Décoloration foliaire. Outre la rhizomanie, les principales sources de décoloration foliaire sont la jaunisse et les carences.

Les atteintes de rhizomanie peuvent se présenter sous forme de trainées alongées ou d'alignements de taches, traduisant le transport par l'eau ou le travail du sol. Ces formes caractéristiques représentent environ $30 \%$ des cas de rhizomanie en 1983 , elles sont beau-

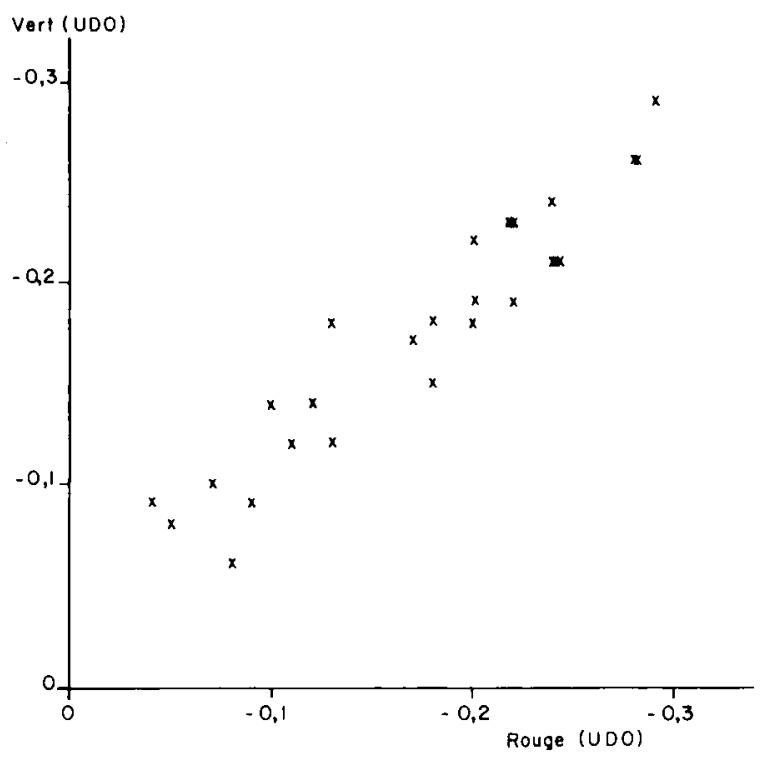

Fig. 4. Variations de densité optique aux transitions entre zones saines et zones atteintes par la rhizomanie mesurées sur les clichés acquis à 1500 m d'altitude (voir Fig. 5). 


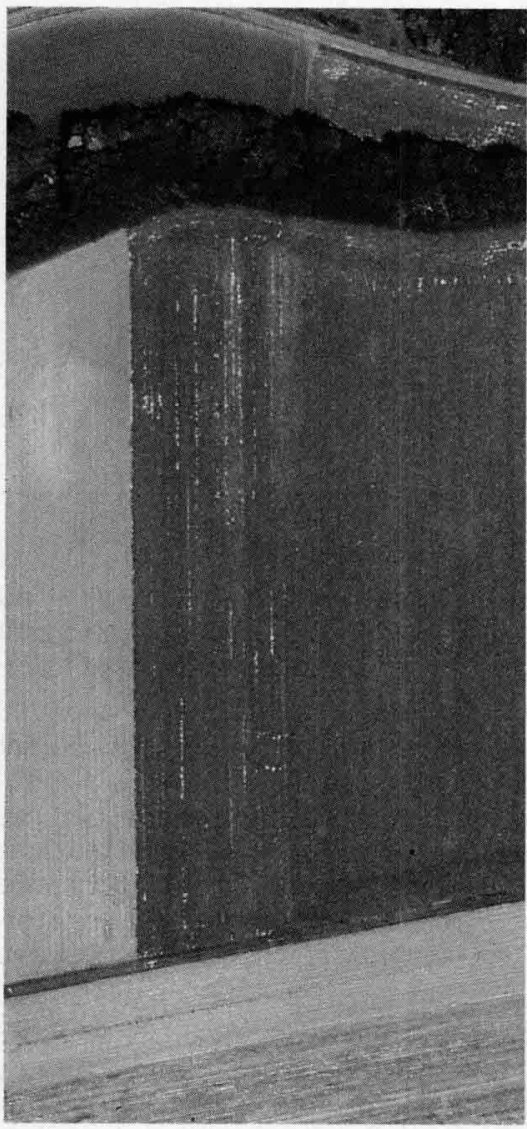

a

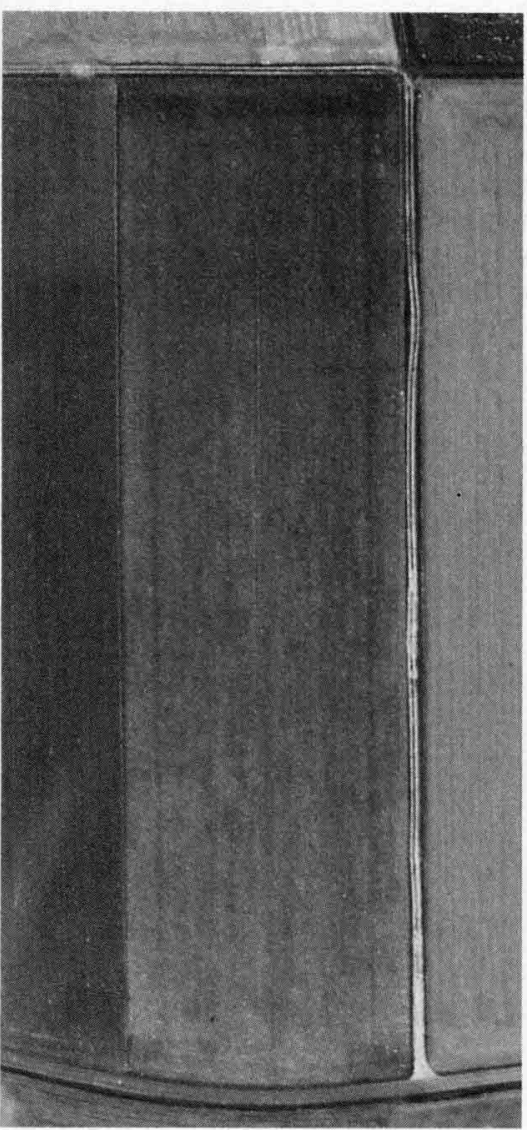

d

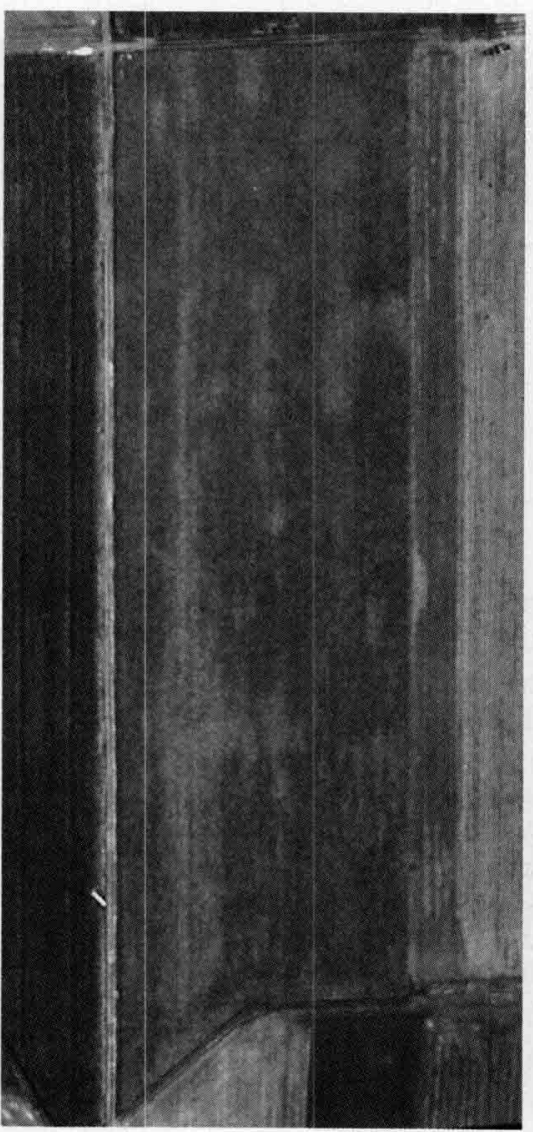

b

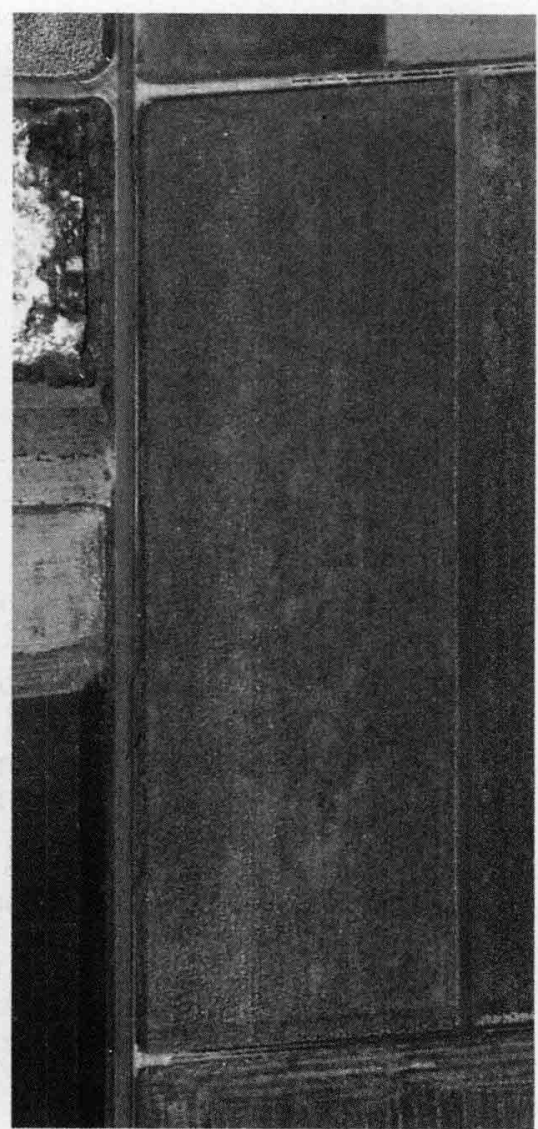

C

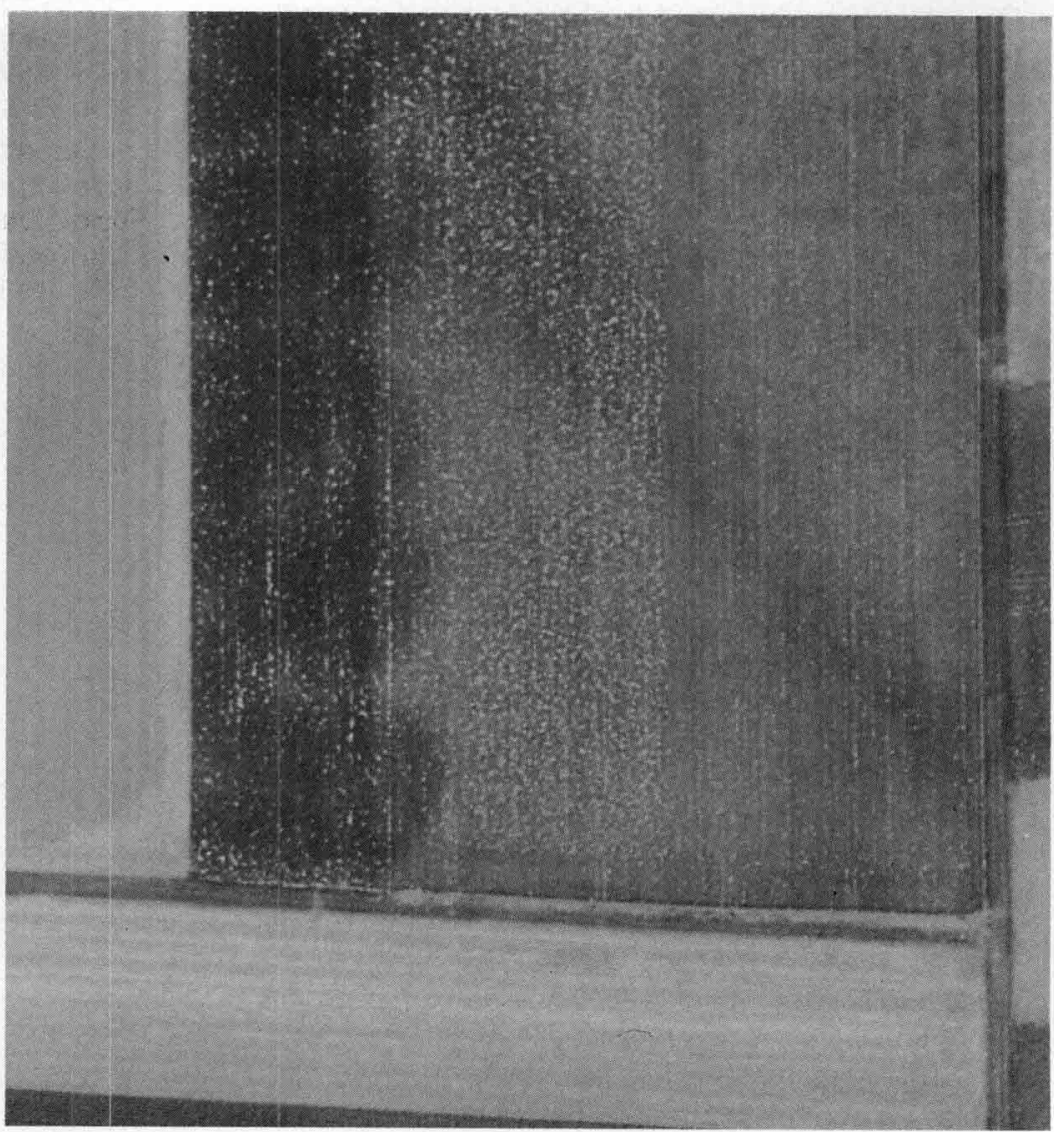

e

Fig. 5. Agrandissements de photographies au 1/10 000. a) Culture avec atteinte localisée; b, c) cultures fortements infestées, montrant les atteintes en traînées, surtout observées en 1983; d) culture fortement infestée, sans forme spécifique des atteintes; e) cultures saines, présentant différents taux de couverture du sol. 
coup plus rares en 1985 , probablement du fait des conditions climatiques défavorables à l'extension de la maladie. La jaunisse se transmet par les vols de pucerons et se présente souvent sous forme de taches dispersées dans les parcelles. Sur notre site d'étude, nous n'avons observé de décolorations fortes liées à des carences que de façon très ponctuelle (lessivage du sol dû à des fuites des systèmes d'irrigation...).

Ces observations nous ont conduit à affecter systématiquement à la rhizomanie les taches présentant une forme caractéristique et en revanche à ne pas prendre en compte les taches isolées et très localisées (dimensions de l'ordre de $10 \mathrm{~m} \times 10 \mathrm{~m}$ ou moins), car les risques de confusion avec un rond de jaunisse ou un problème de carence sont trop élevés; cette limitation ne posait pas de problème en 1983, où les atteintes étaient toujours assez étendues, mais conduit à des omissions en 1985. Dans le cas de décolorations étendues ne présentant pas de formes caractéristiques, il n'est généralement pas possible de distinguer la rhizomanie de la jaunisse. Pour la lutte prophylactique, il est nécessaire d'obtenir une détection aussi élevée que possible, les contrôles de terrain ultérieurs permettant de lever les confusions, ces parcelles ont donc fait l'objet d'un diagnostic positif.

b) Structure du couvert. Le flétrissement des plantes, qui intervient au milieu des journées chaudes, conduit à une augmentation de la réflectance du couvert dans le visible, analogue à celle résultant de décolorations foliaires (Andrieu et Boissard, 1986). Les acquisitions aériennes ont pu être programmées de façon à éviter ce phénomène, à l'exception de la mission du 12 septembre 1985.

Le stress hydrique prolongé provoque une décoloration du feuillage, mais également une forte réduction du taux de couverture du sol, alors que celui-ci est peu affecté par la rhizomanie, du moins en cas d'infestation récente. Compte tenu de l'échelle de prise de vue, la résolution spatiale des clichés est de l'ordre de $\mathbf{3 0}$ $\mathrm{cm}$, ce qui permet d'identifier les parcelles présentant un faible taux de couverture du sol (Fig. 5). Les stress hydriques rencontrés en 1985 ne sont donc pas confondus avec la rhizomanie; toutefois, ils réduisent fortement le taux de détection, car il n'est généralement pas possible de diagnostiquer la rhizomanie lorsque les deux phénomènes interviennent sumultanément.

c) Classification retenue. On a retenue une classification en 2 catégories (sain/atteint). La catégorie "atteint" comprend les parcelles présentant des décolorations caractéristiques de la rhizomanie par leur forme, ainsi que les parcelles présentant simultanément des décolorations marquées et suffisamment étendues (> $100 \mathrm{~m}^{2}$ environ) et un taux de couverture du sol élevé (> 70\% environ). Les autres parcelles sont classées saines (décoloration faible avec absence de formes caractéristiques de la rhizomanie, taches de très petites dimensions, faible taux de couverture du sol).

Les cas douteux ont été classés dans la catégorie "atteint», puisque l'on souhaitait privilégier un taux de détection élevé, plutôt qu'un taux de confusion faible, compte tenu de l'application envisagée.

\section{Résultats}

\section{Cultures irriguées}

Les résultats sont présentés dans le Tableau III.

\section{1) Taux de détection}

En 1983, le taux de détection passe de $53 \%$ le 13 août à $72 \%$ le 7 septembre. L'augmentation est conforme à l'intensification des symptômes observée sur le terrain, et aurait sans doute été plus élevée si la mission de septembre avait pu être effectuée par ciel complètement dégagé. L'utilisation conjointe de deux missions (on affecte à la classe «malade" les parcelles classées malades au cours de l'une ou l'autre des missions) conduit à un taux de détection de $77 \%$ sur culture irriguée.

En 1985 le taux de détection passe de $64 \%$ le 31 août à $45 \%$ le 13 septembre. Cette baisse traduit l'installation du stress hydrique après l'arrêt des irrigations.

\section{2) Taux de confusion}

Le taux de confusion ne peut être évalué correctement en 1983 à cause du faible nombre de parcelles saines. II est d'environ $18 \%$ pour les deux missions de 1985.

Tableau IIl. Evaluation du diagnostic aérien sur parcelles irriguées et non irriguées. Sa: nombre de parcelles saines (d'après l'enquête sol); \%C: taux de confusion, rapport entre le nombre de parcelles saines, mais classées atteintes sur les documents aériens, et le nombre total de parcelles saines (Sa); At: nombre de parcelles atteintes de rhizomanie (d'après l'enquête sol); \%D: taux de détection, rapport entre le nombre de parcelles atteintes et classées atteintes sur les documents aériens et le nombre total de parcelles atteintes (At).

Cultures irriguées

Cultures non irriguées

\begin{tabular}{lrlllllll} 
Date & Sa & $\% C$ & At & $\% D$ & Sa & $\% C$ & At & $\% D$ \\
\hline 13 août 1983 & 7 & 14 & 70 & 53 & 7 & 0 & 16 & 62 \\
7 septembre 1983 & 7 & 0 & 64 & 72 & 7 & 0 & 15 & 40 \\
29 août 1985 & 56 & 18 & 73 & 64 & 84 & 1 & 4 & 0 \\
12 septembre 1985 & 58 & 16 & 77 & 45 & 86 & 0 & 5 & 0
\end{tabular}




\section{Cultures non irriguées}

Les résultats sont présentés dans le Tableau III.

\section{1) Taux de détection}

L'analyse relative à 1983 présente des résultats assez comparables à ceux obtenus en culture irriguée, mais le nombre de parcelles est ici trop faible pour pouvoir conclure précisément.

En 1985, la rhizomanie n'a été identifiée au sol que sur 5 parcelles non irriguées, et n'a jamais pu être mise en évidence sur les clichés; les symptômes foliaires observés au sol sont très ténus, aussi, malgré le faible nombre de points disponibles, on peut conclure que le diagnostic aérien en culture sèche n'est pas possible dans les conditions climatiques rencontrées en 1985.

\section{2) Taux de confusion}

En 1983, on n'observe pas de confusions, toutefois, le nombre de parcelles saines n'est que de 7. L'absence de confusion en 1985 traduit le fait que la plupart des parcelles non irriguées présentent de forts symptômes de stress hydrique, et qu'elles ont en conséquence été classées saines.

\section{Discussion}

Le choix de la photo-interprétation comme méthode d'exploitation des clichés peut être critiqué du fait de la subjectivité inhérente à cette technique; toutefois, il n'y avait pas d'autres moyens d'obtenir dans des délais raisonnables une information à l'échelle du site d'étude. L'objectif poursuivi pose des contraintes importarites sur l'échantillonnage dans le temps (variabilité des symptômes) et dans l'espace (détection d'atteintes très localisées) incompatibles avec les performances actuelles des satellites d'observation de la terre. D'autre part, la numérisation avec une résolution métrique des missions réalisées aurait représenté pour chaque cliché un volume de données équivalent à la moitié d'une scène SPOT. En outre, la photo-interprétation permet d'utiliser des critères de forme que l'on sait très mal prendre en compte numériquement.

Les résultats obtenus portent sur 2 années climatiquement très différentes.

Dans les conditions de forte chaleur et d'humidité rencontrées en 1983, on a obtenu un taux de détection de $72 \%$ en culture irriguée; ce taux aurait été sensiblement amélioré:

- par de meilleures conditions de prise de vue (ennuagement);

- par une date d'acquisition plus tardive, l'extériorisation des symptômes foliaires s'étant accentuée bien après les prises de vue;
- par l'utilisation d'une focale plus longue $(300 \mathrm{~mm})$ pour réduire les effets liés à l'angle de visée.

Dans ces conditions climatiques, l'utilisation de la photographie aérienne en cultures non irriguées semble également envisageable, bien que ces cultures aient été trop peu nombreuses sur notre site d'étude pour que l'on puisse évaluer précisément cette possibilité.

En cas de sécheresse aux mois d'août et septembre, le diagnostic de la rhizomanie par photographie aérienne est impossible en culture sèche, et les résultats obtenus en culture irriguée sont insuffisants pour envisager une application à la lutte prophylactique.

Sur notre site d'étude, représentatif des conditions culturales rencontrées dans les régions betteravières du sud de Paris, l'utilisation de la photographie aérienne comme aide au diagnostic est envisageable les années où les conditions climatiques sont le plus favorables à l'extériorisation de symptômes foliaires; ces conditions sont également celles où la propagation de la maladie est la plus rapide, donc où l'utilisation de la télédétection se justifie le plus pour guider le travail de terrain.

Depuis 1983, la rhizomanie s'est étendue à la majeure partie des régions betteravières en France. Pour évaluer les possibilités pratiques de mise en œuvre de l'approche proposée, plusieurs éléments seraient à préciser:

- le déterminisme des symptômes au champ, en fonction des conditions climatiques et hydriques. Ceci conditionne en effet l'aire géographique où la méthode peut être appliquée et la fréquence des années favorables;

- la possibilité de mettre en ceuvre des procédures d'échantillonnage (transects), dans la mesure où, compte tenu de l'échelle de prise de vue adoptée, une mission aérienne peut couvrir quelques centaines de $\mathrm{km}^{2}$, mais ne permet pas une surveillance exhaustive du territoire;

- l'efficacité économique des mesures prophylactiques, comparée au coût des missions aériennes.

Enfin, pour ce qui concerne le déterminisme de la réflectance des couverts, le principal problème soulevé est celui de la distinction entre les variations de pigmentation foliaire et les variations du taux de couverture du sol. La résolution spatiale des clichés a permis de résoudre en partie cette difficulté par des critères visuels; toutefois ceci implique des acquisitions à relativement basse altitude et conditionne donc directement la dimension des zones couvertes. Nos recherches en cours visent principalement à étudier les possibilités de caractériser la pigmentation foliai- 
re, indépendamment du taux de couverture du sol, en utilisant conjointement les bandes spectrales du visible, du proche et du moyen infrarouge.

\section{Remerciements}

Cette étude a été réalisé dans le cadre d'un contrat entre I'INRA et l'Institut technique de la betterave industrielle. Nous remercions MM. Ciecierski et de Balathier (ITB, Pithiviers), qui ont mis à notre disposition leurs enquêtes de terrain.

\section{Références}

Andrieu B. (1984) Utilisation de données photographiques et radiométriques pour la mise en évidence de la rhizomanie de la betterave. In: Proceedings of the 18th Int. Symp. on Rem. Sens. of Env. Paris. pp. 813823

Andrieu B. \& Boissard P. (1986) Evolution diurne de la réflectance d'un couvert à géométrie variable: la betterave à sucre. Conséquence pour l'interprétation des mesures radiométriques. Bull. Soc. Fr. Photogramm. Télédétection 103, 25-31

Anonyme (1984) Rhizomanie: diagnostic au champ. In: Betteravier français 448, 24-25

Asher M. (1985) Rizomania. The Dutch approach. Br. Sugar Beet Rev. 30-33
Becker F., Nerry F., Ramanantsizehena P. \& Fischer J. (1988) Towards a quantitative use of aerial color infrared films. Photogrammetric Engineering and Remote Sensing. 54, 734-750

Cariolle (1987) Rhizomanie. Mesures de prophylaxie en France et dans d'autres pays. In: C.R. 500 Congrès d'hiver de I'Inst. Int. de Rech. Bett. Bruxelles. pp. 63-78

Hansi (1987) Détection de la rhizomanie en cours de végétation. In: C.R. $50^{\circ}$ Congrès d'hiver de l'Inst. Int. de Rech. Bett. Bruxelles. pp. 195-204

Institut technique français de la betterave industrielle (1983) La Rhizomanie. In : Compte Rendu des travaux effectués en 1983, pp. 194-214

Institut technique français de la betterave industrielle (1985) La Rhizomanie. In : Compte-rendu des travaux effectués en 1985, pp. 177-238

Putz C. \& Richard Molard M. (1984a) La rhizomanie de la betterave, une maladie à virus qui a pris une grande extension en France en 1983. In: C.R. Acad. Agric. de France. 70, 3, pp. 370-378

Putz C.\& Richard Molard M. (1984b) Connaissances actuelles concenant la rhizomanie. In: C.R. Rhizomanie de la Betterave Sucrière. fer colloque international des virologistes, Colmar, France. pp. 5-12

Sanwald E.F. (1981) Laboratory-determined spectral signatures of leaves of healthy and rhizomania diseased sugar beets and disease interpretability from aerial IRC photographs. In: C.R. du fer colloque international sur les signatures spectrales d'objets en télédétection. Avignon, France. pp. 201-208 